\title{
The MECP2/IRAK1 locus modulates SLE risk via epigenetics
}

Polymorphisms in several genetic loci have been linked with systemic lupus erythematosus (SLE), but the mechanisms by which they influence disease development are poorly understood. Now, new research has characterized the effect of a known risk variant in the MECP2/IRAK1 locus.

"The study was done to try to understand how the SLE-risk variant in this genetic locus can increase SLE susceptibility", explains lead author Amr Sawalha. To achieve this aim, the investigators first analysed the expression of IRAK1 and the two splice isoforms of $M E C P 2$ (MECP2A and MECP2B) in primary human $\mathrm{T}$ cells obtained from healthy individuals who were homozygous for either the SLE-risk or the non-risk haplotype. Interestingly, they found that levels of $M E C P 2 B$ mRNA were increased in stimulated T cells with the risk haplotype.

MECP2 is a key regulator of the expression of methylationsensitive genes and also recruits
DNA methyltransferase 1 during DNA replication. Exploring the effects of the MECP2/IRAK1 risk variant on genomewide DNA methylation in stimulated T cells, the investigators found that although the average level of methylation was not altered, $245 \mathrm{CpG}$ sites were differentially methylated in T cells homozygous for the risk haplotype. Many of these sites were in genes associated with antigen presentation (particularly HLA class II genes)

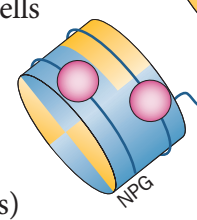
and the interferon response, suggesting that the MECP2/IRAK1 risk variant can affect immune responses through epigenetic mechanisms.

To further investigate the impact of high MECP2 levels, the researchers used transgenic mice overexpressing human MECP2. Gene expression profiling of stimulated $T$ cells from transgenic and control mice identified 109 differentially expressed genes. Notable among these was Eif2c2, which modulates the expression of miR-21, a microRNA known to be upregulated in $\mathrm{CD}^{+} \mathrm{T}$ cells from patients with SLE.

Moreover, pathway analyses suggested that the activity of the IL-6, IL-17 and CD40 signalling pathways, which have important roles in immune responses, might be modified by these expression changes.

But is autoimmunity induced by these effects? Intriguingly, the researchers showed that MECP2-transgenic mice have substantially higher serum levels of antinuclear antibodies than wild-type mice. The next step will be "to look for the effect of this SLE-associated genetic variant in other cell types", proposes Sawalha.

Isabel Woodman

Original article Koelsch, K. A. et al. Functional characterization of the MECP2/IRAK1 lupus risk haplotype in human T cells and a human MECP2 transgenic mouse. J. Autoimmun. doi:10.1016/j.jaut.2012.12.012 